\title{
Almost and Nearly Isosceles Pythagorean Triples
}

\author{
Eunmi Choi \\ Department of Mathematics, Han Nam University, Daejeon, Republic of Korea \\ Correspondence should be addressed to Eunmi Choi; emc@hnu.kr \\ Received 16 June 2016; Accepted 9 August 2016 \\ Academic Editor: Aloys Krieg
}

Copyright (C) 2016 Eunmi Choi. This is an open access article distributed under the Creative Commons Attribution License, which permits unrestricted use, distribution, and reproduction in any medium, provided the original work is properly cited.

This work is about extended pythagorean triples, called NPT, APT, and AI-PT. We generate infinitely many NPTs and APTs and then develop algorithms for infinitely many AI-PTs. Since AI-PT $(a, b, c)$ is of $|a-b|=1$, we ask generally for PT $(a, b, c)$ satisfying $|a-b|=k$ for any $k \in \mathbb{N}$. These triples are solutions of certain diophantine equations.

\section{Introduction}

A pythagorean triple $(\mathrm{PT})$ is an integer solution $(a, b, c)$ satisfying the polynomial $x^{2}+y^{2}=z^{2}$, and it is said to be primitive $(\mathrm{PPT})$ if $\operatorname{gcd}(a, b, c)=1$. There have been many ways for finding solutions of $x^{2}+y^{2}=z^{2}$, and one of the wellknown methods is due to Euclid, BC 300. The investigation of integer solutions of $x^{2}+y^{2}=z^{2}$ has been expanded to various aspects. One direction is to deal with polynomials $x^{2}+y^{2}=$ $z^{2} \pm 1$, where in [1] its integer solutions were called almost pythagorean triple (APT) or nearly pythagorean triple (NPT) depending on the sign \pm . Another side is to study solutions of $x^{2}+y^{2}=z^{2}$ having some special conditions. A solution $(a, b, c)$ is called isosceles if $a=b$. Since there is no isosceles integer solution of $x^{2}+y^{2}=z^{2}$, isosceles-like integer triples $(a, b, c)$ with $|a-b|=1$ were investigated. We shall call the $(a, b, c)$ an almost isosceles pythagorean triple (AI-PT), and typical examples are $(3,4,5)$ and $(20,21,29)$. In literatures [2-4], AI-PT was studied by solving Pell polynomial. And a few others $[5,6]$ used triangular square numbers for finding AI-PT. We note that in some articles AI-PT was called almost isosceles right angled (AIRA) triangle. But in order to emphasize relationships with PT, APT, and NPT in this work, we shall refer to AIRA as AI-PT. APT and NPT were studied in [1] while AI-PT was studied in [2, 4], and so forth, but it seems that no one has asked about their connections.

In this work we generate infinitely many APTs and NPTs and then apply the results in order to develop algorithms for constructing infinitely many AI-PTs. Moreover we study
PTs $(a, b, c)$ satisfying $|a-b|=k$ for any $k \geq 1$. So the study of these triples can be regarded as a research of solving diophantine equations $x^{2}+y^{2}=z^{2} \pm 1$ and $x^{2}-z^{2}=2 y z$.

\section{Almost and Nearly Pythagorean Triples}

APT and NPT, respectively, are integer solutions of $x^{2}+y^{2}=$ $z^{2}+1$ and $x^{2}+y^{2}=z^{2}-1$, respectively. If $(a, b, c)$ is an APT or NPT, so it is $( \pm a, \pm b, \pm c)$ hence we generally assume $a, b, c>0$. Some triples were listed in [1] by experimental observations:

NPT: $(10,50,51),(20,200,201),(30,450,451),(40$, $800,801), \ldots$

APT: $(5,5,7),(4,7,8),(8,9,12),(7,11,13),(11,13$, $17),(10,15,18), \ldots$

Lemma 1 (see [1]). If $(a, b, c)$ is an APT then $\left(2 a c, 2 b c, 2 c^{2}+1\right)$ is a NPT. Conversely if $(a, b, c)$ is a NPT then $\left(2 a^{2}+1,2 a b, 2 a c\right)$ is an APT.

Theorem 2. If $a$ is an even integer then we have the following.

(1) $(a, b, b+1)$ is an APT if $b=a^{2} / 2-1$, while it is a NPT if $b=a^{2} / 2$.

(2) $\left(2 a^{2}+1, a^{3}, a\left(a^{2}+2\right)\right)$ is an APT and $\left(a^{3}, a^{2}\left(a^{2} / 2-\right.\right.$ 1), $\left.a^{4} / 2+1\right)$ is a NPT. 
TABLE 1

\begin{tabular}{lcccc}
\hline$a$ & $\left(a, \frac{a^{2}-2}{2}, \frac{a^{2}}{2}\right)$ & $\left(a^{3}, \frac{a^{2}\left(a^{2}-2\right)}{2}, \frac{a^{4}+2}{2}\right)$ & $\left(a, \frac{a^{2}}{2}, \frac{a^{2}+2}{2}\right)$ & $\left(2 a^{2}+1, a^{3}, a\left(a^{2}+2\right)\right)$ \\
\hline 2 & $(2,1,2)$ & $(8,4,9)$ & $(2,2,3)$ & $(9,8,12)$ \\
6 & $(4,7,8)$ & $(64,112,129)$ & $(4,8,9)$ & $(33,64,72)$ \\
\hline
\end{tabular}

TABLE 2

\begin{tabular}{lcccc}
\hline$a$ & $\mathrm{NPT}\left(a, \frac{a^{2}-24}{10}, \frac{a^{2}+26}{10}\right)$ & $\mathrm{APT}$ & $a$ & $\mathrm{APT}\left(a, \frac{a^{2}-26}{10}, \frac{a^{2}+24}{10}\right)$ \\
\hline 8 & $(8,4,9)$ & $(129,64,144)$ & 14 & $(14,17,22)$ \\
12 & $(12,12,17)$ & $(289,288,408)$ & 16 & $(16,23,28)$ \\
18 & $(18,30,35)$ & $(649,1080,1260)$ & 24 & $(24,55,60)$ \\
\hline
\end{tabular}

Proof. If $c=b+1$ then $c^{2}-b^{2}=2 b+1$. If $b=a^{2} / 2-1$ then $c^{2}-b^{2}=a^{2}-1$, so $(a, b, c)$ is an APT. If $b=a^{2} / 2$ then $c^{2}-b^{2}=a^{2}+1$, so $(a, b, c)$ is a NPT.

Due to Lemma 1 , the NPT $\left(a, a^{2} / 2, a^{2} / 2+1\right)$ yields an APT $\left(2 a^{2}+1, a^{3}, a\left(a^{2}+2\right)\right)$, while the APT $\left(a, a^{2} / 2-1, a^{2} / 2\right)$ provides a NPT $\left(a^{3}, a^{2}\left(a^{2} / 2-1\right), a^{4} / 2+1\right)$ (see Table 1$)$.

Theorem 2 gives infinitely many APTs and NPTs $(a, b, c)$ such that $c-b=1$. Not only this, we can generate APT and NPT $(a, b, c)$ with $c-b=5$.

Theorem 3. (1) If $a \equiv \pm 2(\bmod 10)$ and $b=\left(a^{2}-24\right) / 10$ then $(a, b, b+5)$ is a NPT.

(2) If $a \equiv \pm 4(\bmod 10)$ and $b=\left(a^{2}-26\right) / 10$ then $(a, b, b+$ 5) is an $A P T$.

Proof. The triple $(a, b, b+5)$ is a NPT if $a^{2}+b^{2}=(b+5)^{2}-1$; that is, $b=\left(a^{2}-24\right) / 10$. Since $b>0$ is integer, it must be $a^{2}>24$ and $a^{2} \equiv 24(\bmod 10)$. So $a \equiv \pm 2(\bmod 10)$ with $a \geq 8$. On the other hand $(a, b, b+5)$ is an APT if $a^{2}=10 b+26$; that is, $b=\left(a^{2}-26\right) / 10$. Similar to the above, we have $a^{2}>26$ and $a^{2} \equiv 26 \equiv 4^{2}(\bmod 10)$. Hence $a \equiv \pm 4(\bmod 10)$ with $a \geq 6$.

Theorem 3 together with Lemma 1 yields infinitely many NPTs and APTs (see Table 2).

Though there are APT and NPT $(a, b, b+k)$ with $k=1,5$, no NPT $(a, b, b+k)$ exists if $k=2$ or 3 . In fact if $(a, b, b+2)$ is a NPT then $a^{2}=4 b+3$. But since $a^{2} \equiv 3(\bmod 4)$ is quadratic nonresidue, no solution $a$ exists. Similarly if $k=3$ then $a^{2} \equiv$ $2(\bmod 6)$, so no integer solution $a$.

Theorem 4. For any $k>0, A P T s$ of the form $(a, b, b+k)$ always exist. If $k-1$ is even and square then there exist NPTs of the form $(a, b, b+k)$.

Proof. A triple $(a, b, b+k)$ is an APT if $a^{2}+b^{2}=(b+k)^{2}+1$; that is, $b=\left(a^{2}-k^{2}-1\right) / 2 k$. Then $a^{2} \equiv k^{2}+1 \equiv(k \pm 1)^{2}(\bmod 2 k)$.
Hence if we let $a=2 m k \pm(k \pm 1)$ and $b=2 m(m k \pm k \pm 1)+1$ for $m \in \mathbb{Z}$, then it can be observed that

$$
\begin{aligned}
& (2 m k \pm(k+1), 2 m(m k \pm k \pm 1)+1,2 m(m k \pm k \pm 1) \\
& \quad+1+k)
\end{aligned}
$$

is an APT. In particular, $(k+1,1, k+1)$ is an APT for all $k>0$.

Let $k-1=u^{2}=2 v(u, v \in \mathbb{N})$. For $(a, b, b+k)$ to be a NPT, we must have $a^{2}=2 k b+k^{2}-1$; that is, $b=\left(a^{2}-k^{2}+1\right) / 2 k$. Hence $a^{2} \equiv k^{2}-1(\bmod 2 k)$, so

$$
\begin{aligned}
a^{2} & \equiv(2 v+1)^{2}-1=4 v^{2}+4 v=v(4 v+2)+2 v \equiv 2 v \\
& =u^{2}\left(\bmod 2 u^{2}+2\right) .
\end{aligned}
$$

Write $a^{2}=u^{2}+2 m\left(u^{2}+1\right)$ for $m \in \mathbb{Z}$. Then $b=-u^{2} / 2+m$ and $c=b+k=u^{2} / 2+1+m$. And since $c^{2}-b^{2}-1=k(2 b+k)-1=$ $\left(u^{2}+1\right)(2 m+1)-1=a^{2},(a, b, c)$ is a NPT.

For instance, $(31,43,53),(51,125,135)$ are APTs $(a, b, c)$ with $c-b=10$. Similarly $(34,47,58),(56,137,148)$ are APTs with $c-b=11$. So we have infinitely many APTs $(a, b, c)$ such that $c-b$ is any integer.

On the other hand, consider $k=1,5,17,37$ such that $k-1$ is square. Then Theorem 4 yields NPT $(a, b, b+k)$ satisfying $a^{2}=2 k b+k^{2}-1$ and $b=\left(a^{2}-k^{2}+1\right) / 2 k$. If $k=1$ then $a \equiv 0(\bmod 2)$ and $b=-a^{2} / 2$ yielding that $(a, b, b+1)$ is a NPT; say $(2,2,3)$, and so forth. If $k=5$ then $a \equiv \pm 2(\bmod$ 10) and $b=\left(a^{2}-24\right) / 10$ with $a^{2}>24$ implying that $(a, b, b+$ $5)$ is a NPT; say $(8,4,9)$, and so forth. If $k=17$ then $a \equiv$ $\pm 4(\bmod 34)$ and $b=\left(a^{2}-288\right) / 34$ with $a^{2}>288$ implying that $(a, b, b+17)$ is a NPT; say $(30,18,35)$, and so forth.

Corollary 5. Let $n \equiv 0(\bmod 10)$. If $a=n+10 k$ and $b=$ $n^{2} / 2+10 k(n+5 k)$ for any $k \geq 0$ then $(a, b, b+1)$ is a NPT.

The proof is clear. Thus $(10,50,51),(20,200,201)$, $(30,450,451),(40,800,801), \ldots$ are NPTs, where the list corresponds to the findings in [1]. We now discuss another way to construct NPTs from PPT. 
TABLE 3

\begin{tabular}{|c|c|c|c|c|c|}
\hline$k$ & $a^{2}=k^{2}-1(\bmod 2 k)$ & $a(\bmod 2 k)$ & $a>k$ & $b$ & $(a, b, b+k) \mathrm{NPT}$ \\
\hline \multirow{3}{*}{5} & & & 8 & 4 & $(8,4,9)$ \\
\hline & $a^{2} \equiv 24 \equiv 4(\bmod 10)$ & \pm 2 & 12 & 12 & $(12,12,17)$ \\
\hline & & & 18 & 30 & $(18,30,35)$ \\
\hline \multirow{3}{*}{17} & & & 30 & 18 & $(30,18,35)$ \\
\hline & $a^{2} \equiv 288 \equiv 16(\bmod 34)$ & \pm 4 & 38 & 34 & $(38,34,51)$ \\
\hline & & & 64 & 112 & $(64,112,129)$ \\
\hline \multirow{3}{*}{37} & & & 68 & 44 & $(68,44,81)$ \\
\hline & $a^{2} \equiv 1368 \equiv 36(\bmod 74)$ & \pm 6 & 80 & 68 & $(80,68,105)$ \\
\hline & & & 142 & 254 & $(142,254,291)$ \\
\hline
\end{tabular}

TABLE 4

\begin{tabular}{|c|c|c|c|c|c|}
\hline$z$ & $a^{2}=z^{2}-1(\bmod 2 z)$ & $a(\bmod 2 z)$ & $a>z$ & $b$ & $(a, b, b+z) \mathrm{NPT}$ \\
\hline \multirow{3}{*}{13} & & & 18 & 6 & $(18,6,19)$ \\
\hline & $a^{2} \equiv 168 \equiv 64(\bmod 26)$ & \pm 8 & 34 & 38 & $(34,38,51)$ \\
\hline & & & 44 & 68 & $(44,68,81)$ \\
\hline \multirow{3}{*}{25} & & & 32 & 8 & $(32,8,33)$ \\
\hline & $a^{2} \equiv 624 \equiv 324(\bmod 50)$ & \pm 18 & 68 & 80 & $(68,80,105)$ \\
\hline & & & 82 & 122 & $(82,122,147)$ \\
\hline \multirow{3}{*}{29} & & & 46 & 22 & $(46,22,51)$ \\
\hline & $a^{2} \equiv 840 \equiv 144(\bmod 58)$ & \pm 12 & 70 & 70 & $(70,70,99)$ \\
\hline & & & 128 & 268 & $(128,268,297)$ \\
\hline
\end{tabular}

Theorem 6. For any PPT $(x, y, z)$, there are NPTs $(a, b, c)$ with $c-b=z$.

Proof. The PPT $(x, y, z)$ can be written as $x=u^{2}-v^{2}, y=$ $2 u v$, and $z=u^{2}+v^{2}$ where $u>v>0$ are bipartite and $\operatorname{gcd}(u, v)=1$. Let $u=2 r$ and $v=2 s+1(r, s \in \mathbb{N})$. Clearly $z=u^{2}+v^{2} \equiv 1(\bmod 4)$ and $z$ is odd. For $(a, b, b+z)$ to be a NPT, it satisfies $a^{2}=2 b z+z^{2}-1$ and $b=\left(a^{2}-z^{2}+1\right) / 2 z$. So $a^{2} \equiv z^{2}-1(\bmod 2 z)$ implies $a^{2} \equiv-1(\bmod z)$ and $a^{2} \equiv$ $z^{2}-1 \equiv 0(\bmod 2)$.

If $z$ is a prime then $a^{2} \equiv-1(\bmod z)$ has integer solutions since $z \equiv 1(\bmod 4)$. So with $b=\left(a^{2}-z^{2}+1\right) / 2 z$, there exists a NPT of the form $(a, b, b+z)$. On the other hand if $z=p_{1} \cdots p_{j}\left(p_{i}\right.$ odd primes, $\left.1 \leq i \leq j\right)$, then $z \equiv 1(\bmod 4)$ implies that either every $p_{i} \equiv 1(\bmod 4)$ or there are even number of $p_{i}$ such that $p_{i} \equiv-1(\bmod 4)$ for $1 \leq i \leq j$. Thus Legendre symbol $(-1 / z)$ equals $\left(-1 / p_{1}\right) \cdots\left(-1 / p_{j}\right)=1$, so $a^{2} \equiv-1(\bmod z)$ has integer solutions; hence there is a NPT $(a, b, b+z)$.

The PPT $(x, y, z)$ with $z \leq 40$ are $(3,4,5),(5,12,13)$, $(8,15,17),(7,24,25),(20,21,29)$, and $(12,35,37)$. If $z=$ $5,17,37$ then Table 3 contains the list of NPTs. When $z=$ 13, 25, 29, NPTs are as shown in Table 4.

An APT $(a, b, c)$ satisfying $a=b$ is called an isosceles APT (iso-APT). Analogously an iso-NPT is defined. Though there is no isosceles PT, there are many iso-APTs and iso-NPTs. Indeed iso-APT and iso-NPT $(a, a, c)$ satisfy $a^{2}+a^{2}=c^{2} \pm 1$, so that the pair $(a, c)$ is an integer solution of $2 x^{2}-y^{2}= \pm 1$, which is the Pell polynomial. If $\left(a_{1}, c_{1}\right),\left(a_{2}, c_{2}\right)$ are integer solutions of $2 x^{2}-y^{2}=-1$ then

$$
\begin{aligned}
1 & =\left(2 a_{1}^{2}-c_{1}^{2}\right)\left(2 a_{2}^{2}-c_{2}^{2}\right) \\
& =-2\left(a_{1} c_{2}+a_{2} c_{1}\right)^{2}+\left(2 a_{1} a_{2}+c_{1} c_{2}\right)^{2} .
\end{aligned}
$$

Shows that $\left(a_{1} c_{2}+a_{2} c_{1}, 2 a_{1} a_{2}+c_{1} c_{2}\right)$ satisfies $2 x^{2}-y^{2}=-1$. If $\left(a_{1}, c_{1}\right),\left(a_{2}, c_{2}\right)$ are roots of $2 x^{2}-y^{2}=1$ then $\left(a_{1} c_{2}+\right.$ $\left.a_{2} c_{1}, 2 a_{1} a_{2}+c_{1} c_{2}\right)$ holds $2 x^{2}-y^{2}=-1$.

Let us define a multiplication $\left(a_{1}, c_{1}\right)\left(a_{2}, c_{2}\right)$ by $\left(a_{1} c_{2}+\right.$ $\left.a_{2} c_{1}, 2 a_{1} a_{2}+c_{1} c_{2}\right)$ [7]. For example, a root $(2,3)$ of $2 x^{2}-y^{2}=$ -1 yields $(2,3)^{2}=(12,17)$ satisfying $2 x^{2}-y^{2}=-1$. And a root $(5,7)$ of $2 x^{2}-y^{2}=1$ shows that $(5,7)^{2}=(70,99)$ holds $2 x^{2}-y^{2}=-1$. So the first few nonnegative solutions of $2 x^{2}-y^{2}= \pm 1$ are

$$
\begin{aligned}
& \left\{(0,1)_{-},(1,1)_{+},(2,3)_{-},(5,7)_{+},(12,17)_{-},(29,41)_{+},\right. \\
& \left.(70,99)_{-},(169,239)_{+}, \ldots\right\},
\end{aligned}
$$

where the subscripts,+- indicate solutions of $2 x^{2}-y^{2}= \pm 1$, respectively.

Theorem 7. Let $s_{n}=\left(a_{n}, b_{n}\right)$ for $s_{n+1}=2 s_{n}+s_{n-1}$ with $s_{0}=$ $(0,1), s_{1}=(1,1)$. Then the following hold.

(1) $a_{n+1}=a_{n}+c_{n}$ and $c_{n+1}=a_{n+1}+a_{n}$ and $2 a_{n} a_{n-1}-$ $c_{n} c_{n-1}=(-1)^{n}$. So $S=\left\{s_{n}\right\}_{n \geq 0}$ is a sequence of solutions of $2 x^{2}-y^{2}=(-1)^{n+1}$. 
(2) Let $A=\left(\begin{array}{ll}1 & 2 \\ 1 & 1\end{array}\right)$. Then $s_{n}=s_{n-1} A=s_{0} A^{n}$ by considering $s_{n}$ as a matrix.

(3) Let $S_{+}, S_{-}$be subsets of $S$ consisting of $s_{n+}, s_{n-}$, respectively. If $s_{n} \in S_{ \pm}$then $s_{n+1} \in S_{\mp}$ and $s_{n+2} \in S_{ \pm}$.

Proof. The recurrence $s_{n+1}=2 s_{n}+s_{n-1}$ shows $\left(a_{n+1}, c_{n+1}\right)=$ $\left(2 a_{n}+a_{n-1}, 2 c_{n}+c_{n-1}\right)$. So $a_{2}=2 a_{1}+a_{0}=2=a_{1}+c_{1}$ and $c_{2}=2 c_{1}+c_{0}=3=a_{2}+a_{1}$. Hence if we assume $a_{n}=a_{n-1}+c_{n-1}$ and $c_{n}=a_{n}+a_{n-1}$ then $a_{n+1}=2 a_{n}+a_{n-1}=a_{n}+\left(a_{n}+a_{n-1}\right)=$ $a_{n}+c_{n}$ and $c_{n+1}=\left(2 a_{n}+a_{n-1}\right)+a_{n}=a_{n+1}+a_{n}$.

Clearly $s_{i}=\left(a_{i}, c_{i}\right)(1 \leq i \leq 3)$ are solutions of $2 x^{2}-$ $y^{2}=(-1)^{i+1}$, and $2 a_{i} a_{i-1}-c_{i} c_{i-1}=(-1)^{i}$. If $\left(a_{i}, c_{i}\right)$ satisfies the identities for $i \leq n$ then

$$
\begin{aligned}
2 a_{n+1}^{2}-c_{n+1}^{2}= & 2\left(2 a_{n}+a_{n-1}\right)^{2}-\left(2 c_{n}+c_{n-1}\right)^{2} \\
= & 4\left(2 a_{n}^{2}-c_{n}^{2}\right)+\left(2 a_{n-1}^{2}-c_{n-1}^{2}\right) \\
& +4\left(2 a_{n} a_{n-1}-c_{n} c_{n-1}\right)=(-1)^{n+2}, \\
2 a_{n+1} a_{n}-c_{n+1} c_{n}= & 2\left(2 a_{n}^{2}-c_{n}^{2}\right)+\left(2 a_{n} a_{n-1}-c_{n} c_{n-1}\right) \\
= & (-1)^{n+1} .
\end{aligned}
$$

Now $s_{0} A=(1,1)=s_{1}$ and $s_{1} A=(2,3)=s_{2}=s_{0} A^{2}$. So if we assume $s_{n-1} A=s_{n}=s_{0} A^{n}$ then $s_{0} A^{n+1}=s_{n} A=$ $\left(a_{n}+c_{n}, 2 a_{n}+c_{n}\right)=\left(a_{n+1}, c_{n+1}\right)=s_{n+1}$.

Moreover for $s_{n}=\left(a_{n}, c_{n}\right), s_{n+1}=\left(a_{n}+c_{n}, 2 a_{n}+c_{n}\right)$ satisfies $2\left(a_{n}+c_{n}\right)^{2}-\left(2 a_{n}+c_{n}\right)^{2}=-\left(2 a_{n}^{2}-c_{n}^{2}\right)$. Similarly from $s_{n+2}=$ $s_{n} A^{2}=\left(3 a_{n}+2 c_{n}, 4 a_{n}+3 c_{n}\right)$, we have $2\left(3 a_{n}+2 c_{n}\right)^{2}-\left(4 a_{n}+\right.$ $\left.3 c_{n}\right)^{2}=2 a_{n}^{2}-c_{n}^{2}$. Thus if $s_{n} \in S_{ \pm}$then $s_{n+1} \in S_{\mp}$ and $s_{n+2} \in S_{ \pm}$. This completes the proof.

Corollary 8. Let $\left(a_{1}, a_{1}, c_{1}\right)(i=1,2)$ be either iso-NPTs or iso-APTs. Define a multiplication by $\left(a_{1}, a_{1}, c_{1}\right)\left(a_{2}, a_{2}, c_{2}\right)=$ $\left(a_{1} c_{2}+a_{2} c_{1}, a_{1} c_{2}+a_{2} c_{1}, 2 a_{1} a_{2}+c_{1} c_{2}\right)$. Then the multiplication of iso-NPTs (or iso-APTs) yields an iso-NPT. And the multiplication of iso-APT and iso-NPT yields an iso-APT.

The corollary about iso-APT and iso-NPT follows immediately. Hence sets $S_{-}$and $S_{+}$yield iso-NPTs $\{(2,2,3),(12$, $12,17),(70,70,99),(408,408,577), \ldots\}$ and iso-APTs $\{(1,1$, $1),(5,5,7),(29,29,41),(169,169,239), \ldots\}$.

\section{Almost Isosceles Pythagorean Triple}

The nonexistence of isosceles integer solution of $x^{2}+y^{2}=z^{2}$ intrigues investigations for finding solutions that look more and more like isosceles. By an almost isosceles pythagorean triple (AI-PT), we mean an integer solution $(a, b, c)$ of $x^{2}+$ $y^{2}=z^{2}$ such that $a$ and $b$ differ by only 1 . The triples $(3,4,5),(20,21,29),(119,120,169)$, and $(696,697,985)$ are typical examples of AI-PT.

Let $(a, b, c)$ be an AI-PT with $b=a+1$. If $c=b+k$ for $k \in \mathbb{N}$ then $a^{2}+(a+1)^{2}=(a+1+k)^{2}$, so $a^{2}-2 k a-\left(k^{2}+2 k\right)=0$. The solution $a=k \pm \sqrt{2 k(k+1)}$ is an integer if $2 k(k+1)$ is a perfect square. In fact, if $k=1$ then $2 k(k+1)=4$, so $a=3, b=4$ yields an AI-PT $(3,4,5)$. Let $2 k(k+1)=u^{2}$ for
TABLE 5

\begin{tabular}{cccc}
\hline$n$ & $\left(u_{n}, v_{n}\right)$ & $k_{n}$ & $a_{n}, b_{n}, c_{n}$ \\
\hline 2 & $(2,3)$ & 1 & $3,4,5$ \\
4 & $(12,17)$ & 8 & $20,21,29$ \\
6 & $(70,99)$ & 49 & $119,120,169$ \\
8 & $(408,577)$ & 288 & $696,697,985$ \\
\hline
\end{tabular}

$u \in \mathbb{N}$. Then $u^{2}-2 k^{2}-2 k=0$, so $2 u^{2}-(2 k+1)^{2}=-1$. If $v=2 k+1$ then $2 u^{2}-v^{2}=-1$, so the pairs $(u, v)$ correspond to the pairs $\left(u_{n}, v_{n}\right) \in S_{-}$in Theorem 7 . Hence the set $S_{-}=$ $\{(2,3),(12,17),(70,99), \ldots\}$ together with $k_{n}=\left(v_{n}-1\right) / 2$, $a_{n}=u_{n}+k_{n}, b_{n}=a_{n}+1$, and $c_{n}=b_{n}+k_{n}$ provides Table 5 of $\operatorname{AI-PT}\left(a_{n}, b_{n}, c_{n}\right)$.

Theorem 9. (1) When $\left(u_{n}, v_{n}\right) \in S_{-}$, let $a_{n}=u_{n}+(1 / 2)\left(v_{n}-1\right)$, $b_{n}=u_{n}+(1 / 2)\left(v_{n}+1\right)$, and $c_{n}=u_{n}+v_{n}$. Then $\left(a_{n}, b_{n}, c_{n}\right)$ is an AI-PT with $c_{n}-b_{n}=(1 / 2)\left(v_{n}-1\right)$.

(2) If $\left(u_{n}, v_{n}\right) \in S_{+}$then $\left(a_{n}, b_{n}, c_{n}\right)$ is an AI-PT for $a_{n}=$ $(1 / 2)\left(v_{n}-1\right), b_{n}=(1 / 2)\left(v_{n}+1\right)$, and $c_{n}=u_{n}$.

Proof. If $\left(u_{n}, v_{n}\right) \in S_{-}$then $v_{n}$ is odd since $v_{n}=2 v_{n-1}+v_{n-2}$ in Theorem 7. So if we let $k_{n}=(1 / 2)\left(v_{n}-1\right)$ then $a_{n}=u_{n}+k_{n}$, $b_{n}=a_{n}+1$, and $c_{n}-b_{n}=\left(u_{n}+v_{n}\right)-u_{n}-(1 / 2)\left(v_{n}+1\right)=$ $(1 / 2)\left(v_{n}-1\right)=k_{n}$. Thus

$$
\begin{aligned}
2\left(a_{n}^{2}+b_{n}^{2}\right) & =2\left(2 a_{n}^{2}+2 a_{n}+1\right) \\
& =4\left(u_{n}+\frac{v_{n}-1}{2}\right)^{2}+4\left(u_{n}+\frac{v_{n}-1}{2}\right)+2 \\
& =4 u_{n}^{2}+4 u_{n} v_{n}+v_{n}^{2}+1 \\
& =2 u_{n}^{2}+\left(2 u_{n}^{2}+1\right)+4 u_{n} v_{n}+v_{n}^{2} \\
& =2 u_{n}^{2}+v_{n}^{2}+4 u_{n} v_{n}+v_{n}^{2}=2\left(u_{n}+v_{n}\right)^{2} \\
& =2 c_{n}^{2},
\end{aligned}
$$

since $\left(u_{n}, v_{n}\right) \in S_{-}$satisfies $2 u_{n}^{2}-v_{n}^{2}=-1$. So $\left(a_{n}, b_{n}, c_{n}\right)$ is an AI-PT.

Similarly Theorem 7 says if $\left(u_{n}, v_{n}\right) \in S_{+}$then $\left(u_{n-1}\right.$, $\left.v_{n-1}\right) \in S_{-}$, where

$$
\begin{aligned}
\left(u_{n-1}, v_{n-1}\right) & =\left(u_{n}, v_{n}\right)\left(\begin{array}{ll}
1 & 2 \\
1 & 1
\end{array}\right)^{-1} \\
& =\left(-u_{n}+v_{n}, 2 u_{n}-v_{n}\right) .
\end{aligned}
$$

Hence by letting $a_{n}=-u_{n}+v_{n}+(1 / 2)\left(2 u_{n}-v_{n}-1\right)=(1 / 2)\left(v_{n}-\right.$ $1), b_{n}=-u_{n}+v_{n}+(1 / 2)\left(2 u_{n}-v_{n}+1\right)=(1 / 2)\left(v_{n}+1\right)$, and $c_{n}=-u_{n}+v_{n}+2 u_{n}-v_{n}=u_{n}$, (1) implies that $\left(a_{n}, b_{n}, c_{n}\right)$ is an AI-PT.

Table 5 can be compared to the results in $[2,3]$. A feature here is that we first generate infinitely many isoNPTs $\left(u_{n}, u_{n}, v_{n}\right)$ and then find AI-PTs $\left(u_{n}+\left(v_{n}-1\right) / 2, u_{n}+\right.$ $\left.\left(v_{n}+1\right) / 2, u_{n}+v_{n}\right)$. For instance, $\left(u_{n}, v_{n}\right)=(5,7)$, $(29,41),(169,239)$ in $S_{+}$produce AI-PTs $(3,4,5),(20,21,29)$, 
(119, 120,169), respectively, by Theorem 9. Moreover Pell sequence provides iso-APT, iso-NPT, and AI-PTs.

Theorem 10. Let $\left\{P_{n}\right\}$ be the Pell sequence with $P_{0}=0$ and $P_{1}=1$.

(1) $\left(P_{n}, P_{n}, P_{n-1}+P_{n}\right)$ is an iso-APT if $n$ is odd; otherwise it is an iso-NPT.

(2) $\left((1 / 2)\left(P_{n}+P_{n+1}-1\right),(1 / 2)\left(P_{n}+P_{n+1}+1\right), P_{n+1}\right)$ with even $n$ and $\left((1 / 2)\left(P_{n-1}+P_{n}-1\right),(1 / 2)\left(P_{n-1}+P_{n}+1\right), P_{n}\right)$ with odd $n$ are AI-PTs.

Proof. Let $A=\left(\begin{array}{ll}1 & 2 \\ 1 & 1\end{array}\right)=\left(\begin{array}{cc}P_{0}+P_{1} & 2 P_{1} \\ P_{1} & P_{0}+P_{1}\end{array}\right)$. Then $A^{2}=$ $\left(\begin{array}{cc}P_{1}+P_{2} & 2 P_{2} \\ P_{2} & P_{1}+P_{2}\end{array}\right)$, and it is easy to see $A^{n}=$ $\left(\begin{array}{cc}P_{n-2}+P_{n-1} & 2 P_{n-1} \\ P_{n-1} & P_{n-2}+P_{n-1}\end{array}\right)\left(\begin{array}{ll}1 & 2 \\ 1 & 1\end{array}\right)=\left(\begin{array}{cc}P_{n-1}+P_{n} & 2 P_{n} \\ P_{n} & P_{n-1}+P_{n}\end{array}\right)$ by $P_{n}=2 P_{n-1}+$ $P_{n-2}$. Hence the determinant $(-1)^{n}=\left|A^{n}\right|=\left(P_{n-1}+P_{n}\right)^{2}-2 P_{n}^{2}$ shows (1) due to Theorem 7.

For (2), clearly $s_{n}=s_{0} A^{n}=\left(P_{n}, P_{n-1}+P_{n}\right)$ and $P_{n-1}+P_{n}$ is odd. If $n$ is even then $s_{n} \in S_{-}$, so by Theorem 9 we may let

$$
\begin{aligned}
& a_{n}=P_{n}+\frac{\left(P_{n-1}+P_{n}-1\right)}{2}=\frac{\left(P_{n+1}+P_{n}-1\right)}{2}, \\
& b_{n}=P_{n}+\frac{\left(P_{n-1}+P_{n}+1\right)}{2}=\frac{\left(P_{n+1}+P_{n}+1\right)}{2}, \\
& c_{n}=P_{n}+\left(P_{n-1}+P_{n}\right)=P_{n+1} .
\end{aligned}
$$

So we have an AI-PT $\left(a_{n}, b_{n}, c_{n}\right)$.
Now if $n$ is odd then $s_{n} \in S_{+}$. Again by Theorem 9, we have an AI-PT $\left(a_{n}, b_{n}, c_{n}\right)$ with $a_{n}=(1 / 2)\left(P_{n}+P_{n-1}-1\right), b_{n}=$ $(1 / 2)\left(P_{n}+P_{n-1}+1\right)$, and $c_{n}=P_{n}$.

There are infinitely many iso-APTs and iso-NPTs by means of Pell sequence, where their corresponding pairs are regarded as solutions of $2 x^{2}-y^{2}= \pm 1$. Moreover infinitely many AI-PTs $(a, b, c)$ arose from Pell sequence are solutions of $x^{2}+y^{2}=z^{2}$ with $b-a=1$. Indeed, due to Theorem 10, if $n=9$ then $\left(P_{9}, P_{9}, P_{8}+P_{9}\right)=(985,985,1393)$ satisfies $x^{2}+y^{2}=$ $z^{2}+1$, so it is an iso-APT, while $\left((1 / 2)\left(P_{8}+P_{9}-1\right),(1 / 2)\left(P_{8}+\right.\right.$ $\left.\left.P_{9}+1\right), P_{9}\right)=(696,697,985)$ meets $x^{2}+y^{2}=z^{2}$, so it is an AI-PT. On the other hand if $n=10$ then $\left(P_{10}, P_{10}, P_{9}+\right.$ $\left.P_{10}\right)=(2378,2378,3363)$ is an iso-NPT satisfying $x^{2}+y^{2}=$ $z^{2}-1$, while $\left((1 / 2)\left(P_{10}+P_{11}-1\right),(1 / 2)\left(P_{10}+P_{11}+1\right), P_{11}\right)=$ $(4059,4060,5741)$ is an AI-PT satisfying $x^{2}+y^{2}=z^{2}$.

Besides Pell sequence, Fibonacci sequence is also useful to generate AI-PT. Horadam [8] proved that the four Fibonacci numbers $\left\{F_{n}, F_{n+1}, F_{n+2}, F_{n+3}\right\}$ generate a PT $T_{n}=$ $\left(F_{n} F_{n+3}, 2 F_{n+1} F_{n+2}, F_{n+1}^{2}+F_{n+2}^{2}\right)$. So $\left\{T_{n}\right\}_{n \geq 1}=\{(3,4,5),(5,12$, $13),(16,30,34),(39,80,89),(105,208,233), \ldots\}$ are all PTs. As a generalization, we say a sequence $\left\{f_{n}\right\}$ is Fibonacci type if $f_{n}+f_{n+1}=f_{n+2}$ with any initials $f_{1}$ and $f_{2}$. Clearly $\left\{f_{n}\right\}=\left\{F_{n}\right\}$ if $f_{1}=f_{2}=1$, and any four Fibonacci type numbers $b-a$, $a, b$, and $b+a(b>a>0)$ yield a PT $\left(b^{2}-a^{2}, 2 a b, b^{2}+a^{2}\right)$, Euclid's formula. Let us consider Fibonacci type numbers and their corresponding PTs:

$$
\begin{array}{cc}
\left\{f_{n}\right\}:\{b-a, a, b, b+a\} & \{b+a, b, 2 b+a, 3 b+a\} \\
T_{n}:\left(b^{2}-a^{2}, 2 a b, b^{2}+a^{2}\right) & \left(3 b^{2}+4 a b+a^{2}, 4 b^{2}+2 a b, 5 b^{2}+4 a b+a^{2}\right) .
\end{array}
$$

In particular if $a=1$ and $b=2$, we have

$$
\begin{array}{ccccc}
\left\{f_{n}\right\}:\{1,1,2,3\} & \{3,2,5,7\} & \{7,5,12,17\} & \{17,12,29,41\} & \cdots \\
T_{n}:(3,4,5) & (21,20,29) & (119,120,169) & (697,696,985) & \cdots
\end{array}
$$

And we notice that middle two terms of $\left\{f_{n}\right\}$ are consecutive Pell numbers and the corresponding PT $T_{n}$ are all AI-PT.

Theorem 11. Let $a=P_{n}, b=P_{n+1}$ be Pell numbers. Then the PT generated by four Fibonacci type numbers $b-a, a, b$, and $b+a$ is an AI-PT.

Proof. Consider four Fibonacci type numbers $\{b-a, a, b, b+a\}$ and its generated triple $T_{n}$. We have seen that $T_{n}$ are all AI-PT if $1 \leq n \leq 4$. Now let $T_{n}=\left(x_{n}, y_{n}, z_{n}\right)$ be the PT generated by $\left\{P_{n+1}-P_{n}, P_{n}, P_{n+1}, P_{n+1}+P_{n}\right\}$ for any $n>0$. Since $x_{n}=$ $P_{n+1}^{2}-P_{n}^{2}, y_{n}=2 P_{n} P_{n+1}$, and $z_{n}=P_{n}^{2}+P_{n+1}^{2}$, it is not hard to see that

$$
\begin{aligned}
x_{n}-y_{n} & =\left(P_{n+1}-P_{n}\right)^{2}-2 P_{n}^{2}=\left(P_{n}+P_{n-1}\right)^{2}-2 P_{n}^{2} \\
& =(-1)^{n}
\end{aligned}
$$

due to the determinant of $A^{n}$ in Theorem 10. Thus $T_{n}$ is an AI-PT.

Like for triples $(x, y, z)$ satisfying $|y-x|=1$, it is worth asking for triples $(x, y, z)$ satisfying $|y-x|=k$ for $k \in \mathbb{N}$. For instance, the Fibonacci type numbers $\{1,1,2,3\},\{1,2,3,5\}$, and $\{1,3,4,7\}$ produce PTs $(x, y, z)=(3,4,5),(5,12,13)$, $(7,24,25)$, respectively, where $y-x=1,7,17$.

Theorem 12. For any positive integer $k$, there are infinitely many PTs $(x, y, z)$ satisfying $|y-x|=2 k^{2}-1$.

Proof. We assume $a_{1}=1$ and $b_{1}=k$. Fibonacci type numbers $\left\{a_{1}, b_{1}, a_{1}+b_{1}, a_{1}+2 b_{1}\right\}$ make a PT $T_{1}^{(k)}=(2 k+1,2 k(k+$ $1), 2 k(k+1)+1)$, where the difference $\delta_{1}^{(k)}=\left|y_{1}-x_{1}\right|=2 k^{2}-1$. 
TABLE 6

\begin{tabular}{ccccccccc}
\hline$n$ & $f_{n}{ }^{\prime}$ s & $T_{n}^{(1)}$ with $\delta_{n}^{(1)}=1$ & $f_{n}$ 's & $T_{n}^{(2)}$ with $\delta_{n}^{(2)}=7$ & $f_{n}$ 's & $T_{n}^{(3)}$ with $\delta_{n}^{(3)}=17$ & $f_{n}^{\prime}$ s & $T_{n}^{(4)}$ with $\delta_{n}^{(4)}=31$ \\
\hline 1 & $1,1,2,3$ & $(3,4,5)$ & $1,2,3,5$ & $(5,12,13)$ & $1,3,4,7$ & $(7,24,25)$ & $1,4,5,9$ & $(9,40,41)$ \\
2 & $3,2,5,7$ & $(21,20,29)$ & $5,3,8,11$ & $(55,48,73)$ & $7,4,11,15$ & $(105,88,137)$ & $9,5,14,19$ & $(171,140,221)$ \\
3 & $7,5,12,17$ & $(119,120,169)$ & $11,8,19,27$ & $(297,304,425)$ & $15,11,26,37$ & $(555,572,797)$ & $19,14,33,47$ & $(893,924,1285)$ \\
\hline
\end{tabular}

Secondly if $a_{2}=a_{1}+2 b_{1}, b_{2}=a_{1}+b_{1}$ then Fibonacci type numbers $\left\{a_{2}, b_{2}, a_{2}+b_{2}, a_{2}+2 b_{2}\right\}$ yield a PT $T_{2}^{(k)}=\left(8 k^{2}+10 k+\right.$ $\left.3,6 k^{2}+10 k+4,10 k^{2}+14 k+5\right)$, with $\delta_{2}^{(k)}=\left|y_{2}-x_{2}\right|=2 k^{2}-1$.

Now for any $n>1$, let $a_{n}=a_{n-1}+2 b_{n-1}$ and $b_{n}=a_{n-1}+b_{n-1}$. Assume that the PT $T_{n}^{(k)}=\left(x_{n}, y_{n}, z_{n}\right)=\left(a_{n}\left(a_{n}+2 b_{n}\right), 2 b_{n}\left(a_{n}+\right.\right.$ $\left.\left.b_{n}\right), b_{n}^{2}+\left(a_{n}+b_{n}\right)^{2}\right)$ generated by Fibonacci type numbers $\left\{a_{n}, b_{n}, a_{n}+b_{n}, a_{n}+2 b_{n}\right\}$ satisfies $\delta_{n}^{(k)}=\left|2 k^{2}-1\right|$. Then the next PT $T_{n+1}^{(k)}$ generated by $\left\{a_{n+1}, b_{n+1}, a_{n+1}+b_{n+1}, a_{n+1}+2 b_{n+1}\right\}$ forms

$$
\begin{aligned}
& T_{n+1}^{(k)}=\left(a_{n+1}\left(a_{n+1}+2 b_{n+1}\right), 2 b_{n+1}\left(a_{n+1}+b_{n+1}\right), b_{n+1}^{2}\right. \\
& \left.\quad+\left(a_{n+1}+b_{n+1}\right)^{2}\right) .
\end{aligned}
$$

And we also have

$$
\begin{aligned}
\delta_{n+1}^{(k)} & =\left|y_{n+1}-x_{n+1}\right| \\
& =\left|2\left(a_{n}+b_{n}\right)\left(2 a_{n}+3 b_{n}\right)-\left(a_{n}+2 b_{n}\right)\left(3 a_{n}+4 b_{n}\right)\right| \\
& =\left|a_{n}^{2}-2 b_{n}^{2}\right|=\left|2 b_{n}\left(a_{n}+b_{n}\right)-a_{n}\left(a_{n}+2 b_{n}\right)\right| \\
& =\left|\delta_{n}^{(k)}\right|=\left|2 k^{2}-1\right| .
\end{aligned}
$$

So we have infinitely many PTs $\left(x_{n}, y_{n}, z_{n}\right)$ such that $\mid y_{n}-$ $x_{n} \mid=2 k^{2}-1$.

If $a_{1}=1, a_{2}=k(1 \leq k \leq 4)$ then $T_{1}^{(k)}$ with $\delta_{1}^{(k)}=\mid 2 k^{2}-$ $1 \mid$ are $\{(3,4,5),(5,12,13),(7,24,25),(9,40,41)\} . T_{n}^{(k)}(1 \leq$ $n, k \leq 4)$ with $\delta_{n}^{(k)}=\left|2 k^{2}-1\right|$ are as shown in Table 6 .

\section{Competing Interests}

The author declares that there is no conflict of interests regarding the publication of this paper.

\section{Acknowledgments}

This work was supported by 2016 HanNam University Research Fund.

\section{References}

[1] O. Frink, "Almost pythagorean triples," Mathematics Magazine, vol. 60, no. 4, pp. 234-236, 1987.

[2] C. C. Chen and T. A. Peng, "Classroom note. Almost isoceles right angled triangle," Australasian Journal of Combinatorics, vol. 11, pp. 263-267, 1995.

[3] R. H. Dye and R. W. Nickalls, "82.9 A new algorithm for generating pythagorean triples," The Mathematical Gazette, vol. 82, no. 493, pp. 86-91, 1998.
[4] T. W. Forget and T. A. Larkin, "Pythagorean triads of the form $x, x+1, z$ described by recurrence sequences," The Fibonacci Quarterly, vol. 6, no. 3, pp. 94-104, 1968.

[5] G. Hatch, "Pythagorean triples and triangular square numbers," The Mathematical Gazette, vol. 79, no. 484, pp. 51-55, 1995.

[6] M. A. Nyblom, "A note on the set of almost-isosceles rightangled triangles," The Fibonacci Quarterly, vol. 36, no. 4, pp. 319322, 1998.

[7] D. Burton, Elementary Number Theory, McGraw Hill Education, 2010.

[8] A. F. Horadam, "Fibonacci number triples," The American Mathematical Monthly, vol. 68, pp. 751-753, 1961. 


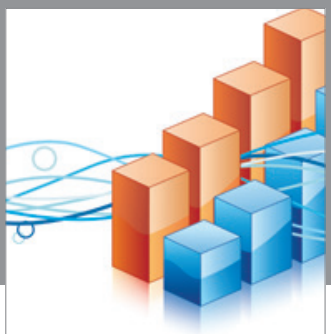

Advances in

Operations Research

vatem alat4

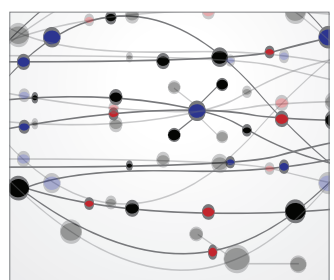

\section{The Scientific} World Journal
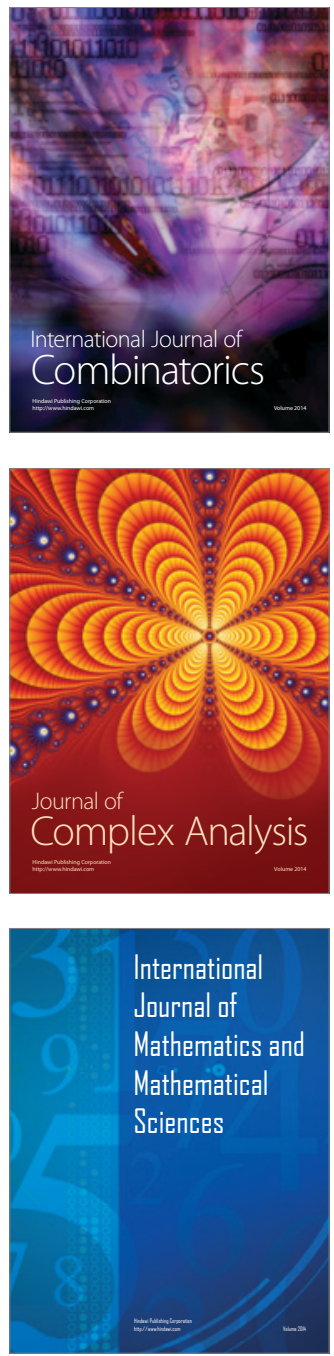
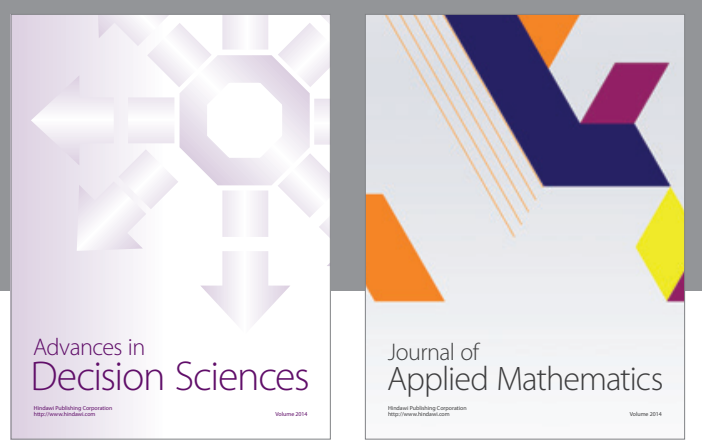

Algebra

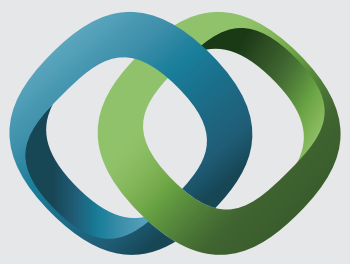

\section{Hindawi}

Submit your manuscripts at

http://www.hindawi.com
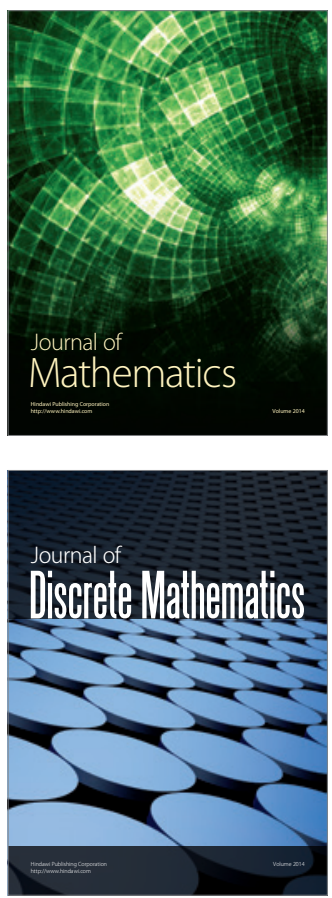

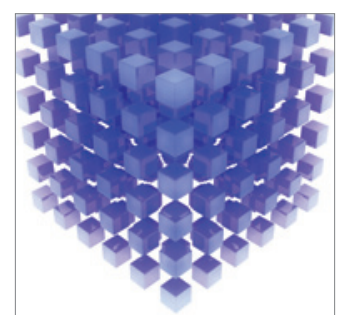

Mathematical Problems in Engineering
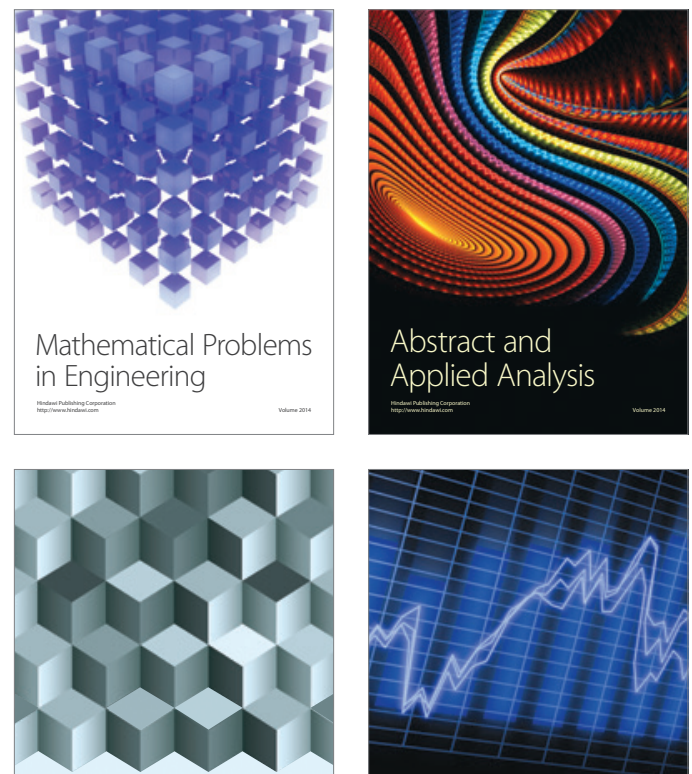

Journal of

Function Spaces

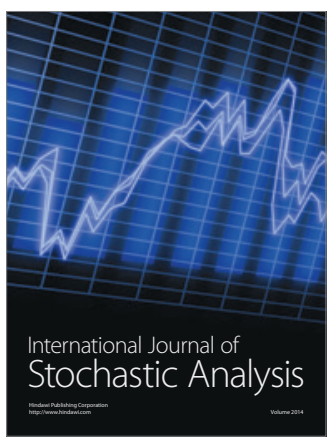

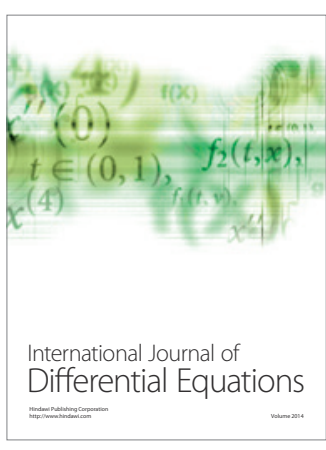
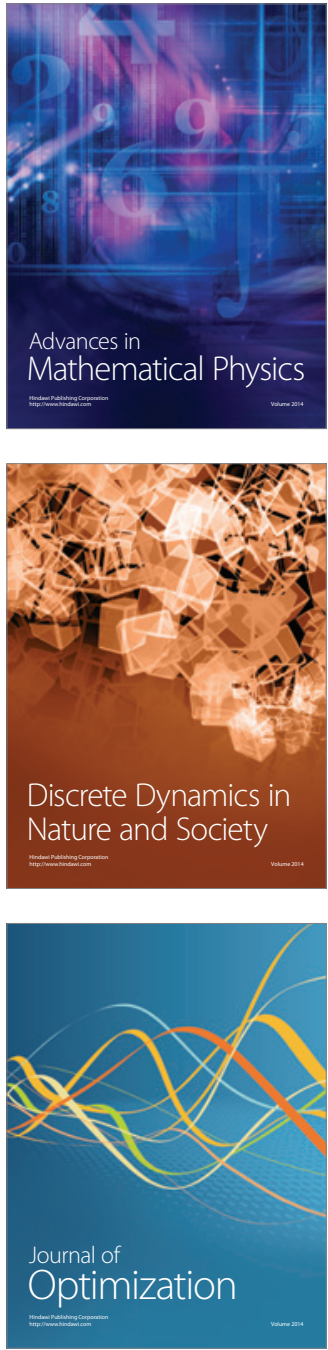